Niayesh Mohebbi ${ }^{1}$, Atefeh Abedini ${ }^{2}$, Reza Lashgari ${ }^{3}$, Fatemeh Razavi ${ }^{2}$, Mohammad Varahram ${ }^{4}$, Arda Kiani $^{5}$

'Clinical Pharmacy Department, Tehran University of Medical Sciences, Tehran, Iran

${ }^{2}$ Chronic Respiratory Diseases Research Center, National Research Institute of Tuberculosis and Lung Diseases, Shahid Beheshti University of Medical Sciences, Tehran, Iran

${ }^{3}$ Institute of Medical Science and Technology, Shahid Beheshti University, Tehran, Iran

${ }^{4}$ Mycobacteriology Research Centre, National Research Institute of Tuberculosis and Lung Disease, Masih Daneshvari Hospital, Shahid Beheshti University of Medical Sciences, Tehran, Iran

${ }^{5}$ Tracheal Diseases Research Center, National Research Institute of Tuberculosis and Lung Diseases, Shahid Beheshti University of Medical Sciences, Tehran, Iran

\title{
Vascular microthrombosis associated with increased interleukin-6. A severe acute respiratory distress syndrome in COVID-19 patients treated with tocilizumab
}

\section{To the Editor}

Thrombosis is an important pathology in the deterioration of COVID-19 patient [1-3]. In the course of the COVID-19 outbreak at the Masih Daneshvari hospital in Iran, we observed different cases of cerebrovascular thrombosis, deep vein thrombosis, and cases of pulmonary microthrombosis despite therapeutic dosing with enoxaparin. These thrombotic cases were detected by transbronchial lung biopsy mostly in COVID-19 infected patients with high interleukin-6 (IL-6) levels. This proposes that respiratory failure and hypoxemia might be associated with microthrombosis in the small vessels of the pulmonary tract. Moreover, we detected monocytosis accompanied by lymphopenia in most of the cases. Monocytosis can additionally potentiate the activation of coagulation pathways through tissue factor release [4-6].

As a matter of fact, IL-6 is a master influencer in inflammatory cytokine drama and correlates with a macrophage activation syndrome that presents in severe COVID-19 cases [7].

Besides all of the proinflammatory characteristics of IL-6, we want to point out its probable role in thrombosis. IL-6 inhibits ADAMTS-13 activity which causes less cleavage of ultra large von Willebrand factors (ULVWF) and results in a hypercoagulative state $[4,8]$. Furthermore, IL-6 stimulates a systemic procoagulant effect by raising the levels of fibrinogen, plasminogen activator inhibitor-1, and C-reactive protein [5].

Seeing as the severe acute respiratory syndrome coronavirus 2 (SARS-CoV-2) disease does not yet have satisfactory treatment options, all health care systems are trying various approaches to save their patients. Tocilizumab, an IL-6 receptor antagonist, is one of the therapeutic strategies in severe cases with increased levels of IL-6 that can be used in order to control this cytokine release syndrome [7]. Surprisingly, however, we detected an increased occurrence in thrombotic events in patients who received tocilizumab. In spite of tocilizumab's mechanism of action, there was evidence of increased levels of IL-6 after treatment with tocilizumab, which could be a possible explanation for the thrombosis [9]. This rise in IL-6 level after tocilizumab administration was seen in all of the cases in our center.

Consequently, we recommend measuring the level of IL-6 following tocilizumab administration. In the case of an increased level, hemoperfusion with cytokine-absorbing columns and continuous renal replacement therapies are suggested in order to decrease the risk of throm-

Address for correspondence: Arda Kiani, Tracheal Diseases Research Center, National Research Institute of Tuberculosis and Lung Diseases,

Shahid Beheshti University of Medical Sciences, Tehran, Iran; e-mail: ardakiani@sbmu.ac.ir

DOI: 10.5603/ARM.a2020.0123

Received: 05.06 .2020

Copyright (C) 2020 PTChP

ISSN 2451-4934 
bosis. Although, plasmapheresis removes IL-6, it may also remove tocilizumab as well, but it can be an alternative method if the preferred one is not available.

All possible approaches with the potential to decrease IL-6 levels or negate IL-6's effects can be considered for research. For instance, based on evidence in relapsing thrombotic thrombocytopenic purpura, $\mathrm{N}$-acetylcysteine may be another option for the prevention of thrombosis by reducing the size and activity of ULVWF [10].

\section{Conflict of interest}

None declared.

\section{References:}

1. Bikdeli B, Madhavan MV, Jimenez D, et al. COVID-19 and thrombotic or thromboembolic disease: implications for prevention, antithrombotic therapy, and follow-up: JACC state-ofthe-art review. J Am Coll Cardiol. 2020; 75(23): 2950-2973, doi: 10.1016/j.jacc.2020.04.031, indexed in Pubmed: 32311448.

2. Leisman DE, Deutschman CS, Legrand M. Facing COVID-19 in the ICU: vascular dysfunction, thrombosis, and dysregulated inflammation. Intensive Care Med. 2020; 46(6): 11051108, doi: 10.1007/s00134-020-06059-6, indexed in Pubmed: 32347323.
3. Sungurlu S, Kuppy J, Balk RA. Role of antithrombin III and tissue factor pathway in the pathogenesis of sepsis. Crit Care Clin. 2020; 36(2): 255-265, doi: 10.1016/i.ccc.2019.12.002, indexed in Pubmed: 32172812.

4. Lee DD, Schwarz MA. Cell-Cell communication breakdown and endothelial dysfunction. Crit Care Clin. 2020; 36(2): 189-200, doi: 10.1016/j.ccc.2019.11.001, indexed in Pubmed: 32172808 .

5. Libby P, Simon DI. Inflammation and thrombosis: the clot thickens. Circulation. 2001; 103(13): 1718-1720, doi: 10.1161/01.cir.103.13.1718, indexed in Pubmed: 11282900.

6. Toussaint S, Gerlach H. Activated protein C for sepsis. N Engl J Med. 2009; 361(27): 2646-2652, doi: 10.1056/NEJMct0808063, indexed in Pubmed: 20042756.

7. McGonagle D, Sharif K, O’Regan A, et al. The role of cytokines including interleukin-6 in COVID-19 induced pneumonia and macrophage activation syndrome-like disease. Autoimmun Rev. 2020; 19(6): 102537, doi: 10.1016/j.autrev.2020.102537, indexed in Pubmed: 32251717.

8. Bernardo A, Ball C, Nolasco L, et al. Effects of inflammatory cytokines on the release and cleavage of the endothelial cell-derived ultralarge von Willebrand factor multimers under flow. Blood. 2004; 104(1): 100-106, doi: 10.1182/ blood-2004-01-0107, indexed in Pubmed: 15026315.

9. Nishimoto N, Terao K, Mima T, et al. Mechanisms and pathologic significances in increase in serum interleukin-6 (IL-6) and soluble IL-6 receptor after administration of an anti-IL-6 receptor antibody, tocilizumab, in patients with rheumatoid arthritis and Castleman disease. Blood. 2008; 112(10): 3959-3964, doi: 10.1182/blood-2008-05-155846, indexed in Pubmed: 18784373.

10. Cabanillas G, Popescu-Martinez A. N-Acetylcysteine for relapsing thrombotic thrombocytopenic purpura: more evidence of a promising drug. Am J Ther. 2016; 23(5): e1277-e1279, doi: 10.1097/MJT.0000000000000386, indexed in Pubmed: $\underline{26720166 .}$. 